\title{
Quantitative Imaging of Estrogen Receptor Expression in Breast Cancer with PET and ${ }^{18}$ F-Fluoroestradiol
}

Lanell M. Peterson ${ }^{1}$, David A. Mankoff ${ }^{1}$, Thomas Lawton ${ }^{2}$, Kevin Yagle ${ }^{1}$, Erin K. Schubert ${ }^{1}$, Svetlana Stekhova ${ }^{1}$, Allen Gown ${ }^{3}$, Jeanne M. Link ${ }^{1}$, Timothy Tewson ${ }^{4}$, and Kenneth A. Krohn ${ }^{1}$

${ }^{1}$ Department of Radiology, University of Washington/Seattle Cancer Care Alliance, Seattle, Washington; ${ }^{2}$ Department of Pathology, University of Washington/Seattle Cancer Care Alliance, Seattle, Washington; ${ }^{3}$ Phenopath Laboratories, Seattle, Washington; and ${ }^{4}$ Department of Nuclear Medicine, University of Iowa, Iowa City, Iowa

The PET compound ${ }^{18} \mathrm{~F}$-fluoroestradiol ( ${ }^{18} \mathrm{~F}-\mathrm{FES}$ ) has been developed and tested as an agent for the imaging of estrogen receptor (ER) expression in vivo. ${ }^{18} \mathrm{~F}-\mathrm{FES}$ uptake has been shown to correlate with ER expression assayed in vitro by radioligand binding; however, immunohistochemistry (IHC) rather than radioligand binding is used most often to measure ER expression in clinical practice. We therefore compared ${ }^{18} \mathrm{~F}$-FES uptake with ER expression assayed in vitro by IHC with both qualitative and semiquantitative measures. Methods: Seventeen patients with primary or metastatic breast cancer were studied with dynamic ${ }^{18} \mathrm{~F}-\mathrm{FES} \mathrm{PET}$; cancer tissue samples, collected close to the time of imaging, were assayed for ER expression by IHC. For each tumor, partial-volume-corrected measures of ${ }^{18} \mathrm{~F}$-FES uptake were compared with ER expression measured by 3 different ER scoring methods: qualitative scoring $(0-3+)$, the Allred score (0-10), and a computerized IHC index. Results: There was excellent agreement $(r=0.99)$ between observers using $\mathrm{IHC}$ as well as the different methods of measuring ER content $(P<0.001)$. ER-negative tumors had ${ }^{18} \mathrm{~F}-\mathrm{FES}$ partial-volumecorrected standardized uptake values of less than 1.0, whereas ER-positive tumors had values above 1.1. Correlation coefficients for the different measures of ER content and the different measures of ${ }^{18} \mathrm{~F}$-FES uptake ranged from 0.57 to 0.73 , with the best correlation being between the computerized IHC index and ${ }^{18} \mathrm{~F}-\mathrm{FES}$ partial-volume-corrected standardized uptake values. Conclusion: Our results showed good agreement between ${ }^{18} \mathrm{~F}$-FES PET and ER expression measured by IHC. ${ }^{18} \mathrm{~F}-$ FES imaging may be a useful tool for aiding in the assessment of ER status, especially in patients with multiple tumors or for tumors that are difficult to biopsy.

Key Words: estrogen receptor; breast cancer; ${ }^{18} \mathrm{~F}-\mathrm{FES}$ PET; IHC; tracer uptake

J Nucl Med 2008; 49:367-374

DOI: 10.2967/jnumed.107.047506

Received Sep. 21, 2007; revision accepted Dec. 4, 2007.

For correspondence or reprints contact: David A. Mankoff, MD, PhD, Department of Radiology, Seattle Cancer Care Alliance, 825 Eastlake Ave. East, Mail Stop 62-600, Seattle, WA 98109.

E-mail: dam@u.washington.edu

COPYRIGHT @ 2008 by the Society of Nuclear Medicine, Inc.
B reast cancer is the most common nondermatologic cancer and the second leading cause of cancer death in women (1). Advances in the understanding of breast cancer biology have led to the development of a wide array of systemic therapies for patients with breast cancer relapse. One major therapeutic option is hormonal therapy, directed at interrupting the estrogen-based signal that stimulates growth in the majority of breast cancers (2). The level of estrogen receptor (ER) expression in breast cancer has been shown to carry important prognostic information and also to predict the likelihood of a response to hormonal therapy (3-5). ER expression is measured in newly diagnosed breast cancer, as a matter of clinical routine, most commonly by immunohistochemistry (IHC) of fixed tissue $(6,7)$. Comparisons of IHC and the previously used radioligand-binding assay for measuring ER expression have shown the practicality and predictive capability of IHC (6-9), and most centers now use IHC rather than radioligand binding as their routine assay for ER expression.

Studies have shown that PET with ${ }^{18} \mathrm{~F}$-fluoroestradiol $\left({ }^{18} \mathrm{~F}\right.$ FES) can be used to noninvasively assess regional ER expression $(10,11)$ and has the potential to overcome the sampling errors that arise from disease heterogeneity and that are associated with in vitro assays for cancer. PET can simultaneously measure the in vivo delivery and binding of estrogens and thus ER expression at multiple tumor sites. Earlier studies showed that ${ }^{18}$ F-FES uptake at a tumor site correlates with ER expression assayed in vitro by radioligand binding (11) and that the level of uptake predicts the likelihood of a response to tamoxifen and aromatase inhibitor treatment (12-14). In this study, we extended this work to compare ${ }^{18} \mathrm{~F}$-FES uptake with ER expression assayed in vitro by IHC, the method currently used for making clinical therapy decisions at most centers $(9,15,16)$. We also report alternate measures of ${ }^{18} \mathrm{~F}$-FES uptake that account for ${ }^{18} \mathrm{~F}$ FES blood clearance to account for rapid ${ }^{18} \mathrm{~F}-\mathrm{FES}$ metabolism and variable ${ }^{18}$ F-FES blood levels (17). Our hypotheses were that ${ }^{18} \mathrm{~F}$-FES uptake correlates with ER expression 
measured in vitro as a semiquantitative index of ER immunostaining and that measures of ${ }^{18} \mathrm{~F}$-FES uptake accounting for blood clearance or metabolism may improve the correlation with in vitro assays of ER expression.

\section{MATERIALS AND METHODS}

Seventeen patients ( 16 women and 1 man) with biopsy-proven or clinically assessed breast cancer were enrolled in the study. All patients with newly diagnosed or recurrent breast cancer and at least one tumor site that had a diameter of $1.5 \mathrm{~cm}$ or greater and that had been sampled or was being considered for sampling were eligible for the study. All patients underwent either core needle or surgical biopsy or removal of their tumors close to the time of the ${ }^{18} \mathrm{~F}-\mathrm{FES}$ PET scan. For patients undergoing surgical biopsy or mastectomy $(n=10)$, PET was performed before surgery in all but 1 patient to avoid the confounding effects of recent surgery on tracer delivery. In the one exception, there was known extensive residual disease because the patient's surgery was discontinued during an intraoperative consultation. For patients undergoing core needle biopsy, our experience has shown little influence on subsequent PET studies $(18,19)$; therefore, they were allowed in this protocol $(n=7 ; 6$ needle biopsies before the ${ }^{18}$ F-FES scan (21-2 d before) and 1 needle biopsy the day after the ${ }^{18}$ F-FES scan). All but 2 patients were not receiving treatment for breast cancer at the time of the PET studies and biopsies. One patient received short-term exposure to an aromatase inhibitor, and one patient had $1 \mathrm{wk}$ of chemotherapy and radiation therapy between the ${ }^{18}$ F-FES scan and the biopsy. Six patients with earlier tamoxifen use were studied at least 2 mo after discontinuing tamoxifen to avoid receptor-binding antagonism by tamoxifen or tamoxifen metabolites. There was no required minimum time for not receiving therapies not known to bind to the ER or to block estradiol-ER binding. One patient had been on the antiestrogen fulvestrant but had stopped the treatment 2 mo before the ${ }^{18} \mathrm{~F}-\mathrm{FES}$ scan. One patient had stopped aromatase inhibitor therapy $2 \mathrm{~d}$ before the ${ }^{18} \mathrm{~F}$-FES scan. One patient was on hormone replacement therapy up to $1 \mathrm{wk}$ before the ${ }^{18} \mathrm{~F}-\mathrm{FES}$ scan. All patients met eligibility requirements and signed informed consent forms in accordance with University of Washington human subject guidelines. The protocols were approved by the University of Washington Human Subjects Committee and Radioactive Drug Research Committee.

An additional 21 patients who underwent both tissue sampling and an ${ }^{18} \mathrm{~F}$-FES scan were considered for inclusion. They were excluded from the analysis because the tissue samples were outside the field of view of the ${ }^{18} \mathrm{~F}-\mathrm{FES}$ study $(n=4)$, the tumor was too small $(<1.5 \mathrm{~cm})$ to accurately quantify with PET $(n=2)$, no tumors were present in the tissue samples $(n=4)$, the patients had intervening breast cancer treatment of several weeks or more between the scan and the biopsy $(n=8)$, the specimens were from lung cancer patients with earlier breast cancer and possible lung metastases $(n=2)$, or the specimen was a vertebral lesion $(n=1)$ with too few tumor cells present for a histologic assay of ER expression.

Tissue was sampled from the breast $(n=12)$, lymph nodes $(n=1)$, axilla $(n=2)$, sternum $(n=1)$, and chest wall $(n=1)$. The mean tumor size was $3.3 \mathrm{~cm}$ (range $=1.5-12 \mathrm{~cm}$ ).

\section{${ }^{18}$ F-FES Synthesis and Quality Control}

Reagents and solvents for synthesis and purification were obtained from Aldrich Chemical Co. or from U.S. Pharmacopeiaapproved suppliers and were used without further purification unless otherwise noted. ${ }^{18} \mathrm{~F}$ was produced by use of either a Siemens Eclipse or a Scanditronix MC-50 cyclotron. ${ }^{18} \mathrm{~F}-\mathrm{FES}$ was prepared according to modifications of published methods (20). A typical injection consisted of approximately $222 \mathrm{MBq}$ of radiopharmaceutical in $20 \mathrm{~mL}$ of isotonic phosphate-buffered saline containing less than $15 \%$ ethanol by volume.

High-performance liquid chromatography-mass spectrometry analysis [Waters 2690 and MicroMass ZMD (ES-)] was performed immediately after each synthesis to evaluate radiochemical and chemical purities and to measure the mass of ${ }^{18} \mathrm{~F}$-FES in the dose. In all instances, the radiochemical purity was found to be $\geq 98 \%$. The specific activity was typically greater than $37 \times 10^{12} \mathrm{~Bq} / \mathrm{mmol}$ at the time of injection. In no instance was more than $5 \mu \mathrm{g}$ of ${ }^{18} \mathrm{~F}-\mathrm{FES}$ injected.

\section{PET}

All patients were positioned supine and imaged with a GE Healthcare Advance PET scanner. Performance details for the tomograph were previously reported $(21,22)$. Calibration of the PET scanner was routinely done by imaging vials containing a known quantity of ${ }^{18} \mathrm{~F}$ assayed in a dose calibrator (model CRC12; Capintec). The vial images were reconstructed with the same reconstruction parameters as those used in the patient studies to allow cross-calibration of $\mathrm{MBq} / \mathrm{mL}$ from region-of-interest (ROI) analysis of the images.

Before PET, patients had intravenous catheters placed for radiopharmaceutical injection and for blood sampling. Using short transmission scans, patients were placed in the tomograph in a position to view a known tumor site for which biopsy results were available for comparison. Chest sites were preferred to enable image-based measurement of the arterial blood clearance curve by use of the left ventricular blood pool (23). A 20- to 25-min transmission scan was performed for attenuation correction. ${ }^{18} \mathrm{~F}$ FES at 125.8-233.1 MBq (3.4-6.3 mCi) (average = 196.1 MBq [5.3 $\mathrm{mCi}$ ) was infused over $2 \mathrm{~min}$ in a volume of $20 \mathrm{~mL}$. Dynamic emission imaging began with the start of infusion and continued for $60 \mathrm{~min}$ with the following time bins: $4 \times 20 \mathrm{~s}, 4 \times 40 \mathrm{~s}, 4 \times 60 \mathrm{~s}, 4 \times$ $180 \mathrm{~s}$, and $8 \times 300 \mathrm{~s}$. Data obtained at 30-60 min were summed to measure the ${ }^{18} \mathrm{~F}$-FES standardized uptake value (SUV) and to aid in drawing ROIs.

\section{Plasma Processing for Metabolite Analysis}

Samples of whole venous blood were collected from patients at 5,20 , and $60 \mathrm{~min}$ after injection. The blood was centrifuged at 3,000 rpm for $5 \mathrm{~min}$, and the plasma was withdrawn. The plasma was used for high-performance liquid chromatography metabolite analysis to determine blood radioactivity present as ${ }^{18} \mathrm{~F}$-FES versus labeled metabolites as previously described (17).

\section{PET Image Analysis}

ROIs were placed over the central portion of the tumor site by reference to an ${ }^{18} \mathrm{~F}$-FDG PET scan, correlative anatomic imaging (mammogram, ultrasound, or CT), and the PET transmission scan. Square ROIs ( $1.5 \mathrm{~cm}$ in diameter) were placed over the center of the tumor site on 3 adjacent imaging planes by use of ${ }^{18} \mathrm{~F}$-FES and ${ }^{18} \mathrm{~F}$ FDG PET images as guides. We used a similar approach in earlier PET studies as a compromise between partial-volume effects seen with larger regions and the high statistical image noise experienced with smaller regions (18). For patients undergoing core needle biopsy, the site of the biopsy was considered in placing the ROIs. Three adjacent $1.5-\mathrm{cm}$ ROIs were placed over the left ventricle to measure the blood clearance curve. We previously showed that this 
approach results in a blood clearance curve comparable to that obtained by arterial blood sampling (24).

The estimated uptake of ${ }^{18} \mathrm{~F}-\mathrm{FES}$ at the tumor site was corrected for partial-volume sampling by use of tumor sizes obtained from the anatomic imaging study (mammogram, ultrasound, or CT) that best depicted the tumor boundaries. As in earlier studies (19), the partialvolume-corrected (PV) uptake was calculated with the following expression:

$$
\mathrm{A}^{\prime}=[(\mathrm{A}-\mathrm{Bck}) / \mathrm{RC}]+\mathrm{Bck},
$$

where $\mathrm{A}^{\prime}$ is the $\mathrm{PV}$ uptake, $\mathrm{A}$ is the uncorrected tumor uptake, Bck is the background uptake obtained from a contralateral normal tissue ROI, and RC is the recovery coefficient. Recovery coefficients, which are equal to measured divided by true activity concentrations, were obtained from an analysis of hot-sphere phantoms with the same ROIs as those used to obtain tumor time-activity curves (25). Note that in Equation 1, when there was no partial-volume count loss $(\mathrm{RC}=1)$, then $\mathrm{A}^{\prime}=\mathrm{A}$. This was the case for tumors larger than $2.5 \mathrm{~cm}$. No tumors were close to the liver, in which uptake could affect the background value.

Tumor ${ }^{18}$ F-FES uptake was quantified by use of 3 different uptake measures: the average SUV of the hottest portion of the tumor (SUV), total flux (flux $x_{\text {tot }}$ ), and flux corrected for labeled metabolites (flux corr $)$. The SUV was calculated by a commonly used formula:

$$
\mathrm{SUV}=\frac{\overline{\mathrm{A}}}{\mathrm{ID} / \mathrm{wt}},
$$

where $\overline{\mathrm{A}}$ is the average tissue uptake at $30-60 \mathrm{~min}$ after injection, ID is the injected dose (in MBq), and wt is the patient weight (in kilograms).

The flux $_{\text {tot }}$ measure of ${ }^{18}$ F-FES uptake, which accounts for variable ${ }^{18} \mathrm{~F}$-FES blood clearance, was defined as follows:

$$
\text { flux }_{\text {tot }}=\frac{\overline{\mathrm{A}}}{\int C_{b} d t},
$$

where $C_{b}$ is the blood clearance curve over time. The flux measure has units of $\mathrm{mL} / \mathrm{min} / \mathrm{g}$ and is similar to the SUV but uses the area under the blood clearance curve as an indicator of tracer availability to the tumor instead of injected dose/patient weight (26). We also calculated the flux measure when the blood clearance curve was corrected for labeled metabolites, as previously described (17); this measure $\left(\right.$ flux $_{\text {corr }}$ ) was defined as follows:

$$
\text { flux }_{\mathrm{corr}}=\frac{\overline{\mathrm{A}}}{\int C_{b_{\mathrm{FES}}} d t},
$$

where $C_{b \mathrm{FES}}$ is the blood clearance curve corrected for labeled metabolites over time.

\section{IHC Analysis}

Tissue blocks from sections of the ${ }^{18} \mathrm{~F}-\mathrm{FES}$-imaged tumors were prepared, sectioned in 4- to 5- $\mu \mathrm{m}$-thick slices, and placed on glass slides. IHC of tumor samples was performed with monoclonal antibody 1D5 (M 7047/JA/03.03.99; Dako), directed against the ER. All reagents were purchased from Dako, and testing was done by use of a Dako autostainer. All samples were processed in a single batch with a positive control. For each sample, a pathologist, unaware of the ${ }^{18} \mathrm{~F}$-FES results, identified 3 areas on each slide for digital photography and storage. All photomicrographs were recorded with the same magnification and lighting to minimize differences. The results of the immunoassay for ER were qualitatively assessed by a pathologist using a scale from 0 to $3+$, with a sample being reported as positive if greater than $5 \%$ of the cells in the sample were positively immunostained. If a discrepancy in the score existed between the original pathology report at the time of the surgery and the score determined from the digital photographs $(n=2)$, then the photographs were reviewed by another pathologist. The samples were also scored by a pathologist using the Allred scoring system, which was recently adopted as the standard method of reporting ER staining at the University of Washington $(7,27)$. Each photomicrograph was evaluated, and the score for the 3 photomicrographs was averaged to produce one value per patient.

In addition, a semiquantitative index of ER staining (IHC index) was calculated by use of a previously described automated image analysis technique and was verified by comparison with a radioligand-binding assay (Photoshop; Adobe Systems) $(28,29)$. In brief, the Photoshop Magic Wand tool was used to identify a representative cell and subsequently cells with a similar intensity. The histogram command was then applied to obtain the mean optical density, which was recorded as the mean value for the tumor. The inverse area was then selected, and the histogram mean density was recorded as the background. The final value recorded was the background minus the mean.

To test the variability of the IHC index, which has not yet been reported, we sent the digitized photographs to 2 independent reviewers for analysis. Each reviewer repeated the procedure 3 times on each of 3 photomicrographs, selecting a different initial cell. The 3 readings on each photomicrograph were averaged, and the averages for the 3 photomicrographs were then averaged to produce one value. The latter values from the reviewers were then averaged to obtain the final quantitative value.

\section{Statistical Analyses}

Although partial-volume correction adds complexity to the estimation of ${ }^{18} \mathrm{~F}$-FES uptake, the intent of our study was to validate ${ }^{18} \mathrm{~F}-\mathrm{FES}$ uptake as a measure of ER expression in comparison with ER expression determined by IHC independent of tumor size. Because of the expected influence of partial volumes for smaller tumors, partial-volume corrections were necessary to accurately measure ${ }^{18} \mathrm{~F}$-FES uptake. Therefore, only PV ${ }^{18} \mathrm{~F}$-FES uptake values were compared with the ER expression results. The measures of ${ }^{18} \mathrm{~F}-$ FES uptake (PV SUV [PVSUV], PV flux tot $_{\text {[PVFES flux }}$ tot, and PV flux $_{\text {corr }}\left[\mathrm{PVFES}\right.$ flux $\left.\mathrm{x}_{\text {corr }}\right]$ ) were compared with the measures of ER expression (qualitative ER scoring, Allred scoring, and IHC), and the results were reported by use of Spearman correlation coefficients $(\rho)$. The correlation between ${ }^{18} \mathrm{~F}$-FES uptake and the semiquantitative IHC index was also evaluated by use of a linear bivariate fit.

\section{RESULTS}

\section{Patient Characteristics}

The average age of the 17 patients was 55 y (range $=39-$ 79 y). Eleven patients $(65 \%)$ were postmenopausal. Six patients $(35 \%)$ had estradiol levels below the detectable level of $20 \mathrm{pg} / \mathrm{mL}$. The average estradiol level for the remaining 11 patients was $63 \mathrm{pg} / \mathrm{mL}$ (range $=26-149 \mathrm{pg} / \mathrm{mL}$ ). Eight 
patients $(47 \%)$ had no therapy before the ${ }^{18} \mathrm{~F}-\mathrm{FES}$ scan. Two patients (12\%) had previously received chemotherapy and radiation therapy. The remaining 7 patients $(41 \%)$ each had previously received one of the following therapies: tamoxifen alone; chemotherapy alone; radiation therapy and tamoxifen; chemotherapy, radiation therapy, and tamoxifen; chemotherapy, tamoxifen, and an aromatase inhibitor; radiation therapy, tamoxifen, and an aromatase inhibitor; or chemotherapy, radiation therapy, tamoxifen, and an aromatase inhibitor.

Two patients had short-term exposure to antitumor agents before the ${ }^{18} \mathrm{~F}$-FES scan and biopsy. One patient began aromatase inhibitor treatment less than 1 mo before the ${ }^{18} \mathrm{~F}$-FES scan and biopsy. One patient received several doses of oral cyclophosphamide and $1 \mathrm{wk}$ of fractionated radiation between the ${ }^{18}$ F-FES scan and surgery.

Blood was collected from all but one patient, for whom PVFES flux corr therefore could not be calculated. Thus, data from 16 patients were included in the PVFES flux ${ }_{\text {corr }}$ analyses, and data from 17 patients were included in all of the other analyses.

\section{IHC Index Observer Variability}

Our analysis of the interobserver variability of the IHC index showed excellent agreement between the 2 observers who used the automated analysis to calculate ER uptake measured by IHC, as shown in Figure 1. The $r$ value was 0.99 $(P<0.001)$, and the average difference between observers was one percentage point (coefficient of variation $=6 \%$ ).

\section{Comparison of ER Analysis Methods}

There was good agreement among all in vitro measures of estrogen content, as shown in Figure 2. The rank correlation coefficients were $0.74(P<0.001)$ when qualitative ER

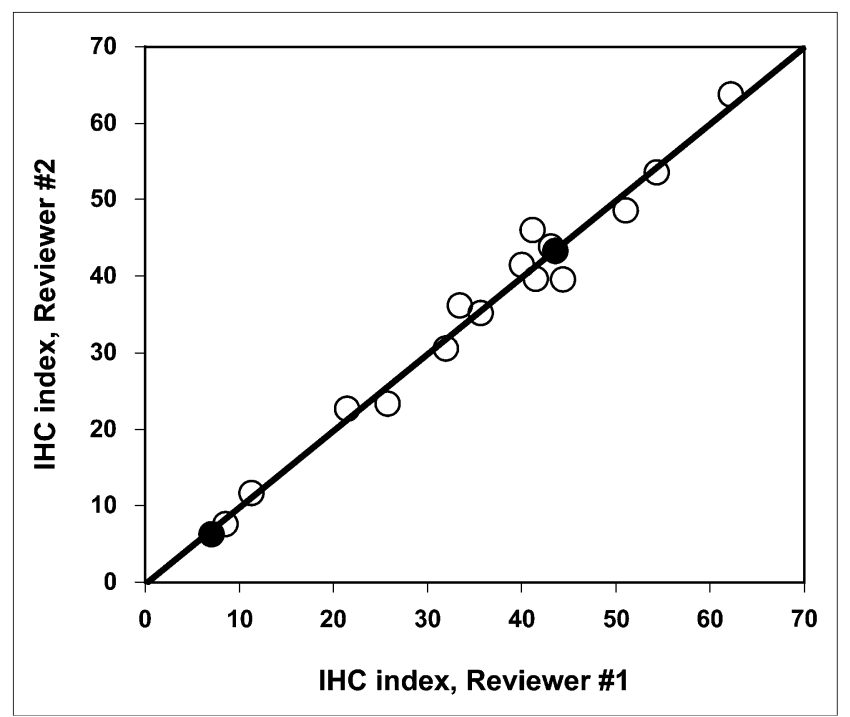

FIGURE 1. Interobserver variability of IHC index determined by Photoshop analysis. Line of identity is also shown. Average difference between observers was $1 \%$. Linear correlation was 0.99. Treated patients are identified by closed circles. uptake (ER positive, $1+$ to $3+$ immunostaining, vs. ER negative) was compared with the Allred scores (Fig. 2A) and $0.73(P<0.001)$ when it was compared with the IHC index (Fig. 2B). For 3 patients, qualitative ER uptake and the Allred scores were both 0 . The correlation coefficient for the Allred scores and the IHC index was $0.81(P<0.001)$ (Fig. 2C).

\section{${ }^{18}$ F-FES Uptake Values and Comparison with IHC Results}

The mean SUV for the 17 patients included in the present study was 1.3 (range $=0.54-5.4)$. When the SUVs were corrected for partial-volume sampling, the mean value changed to 2.0 (range $=0.54-5.6$ ). The means (ranges) for flux measures were as follows: flux ${ }_{\text {tot }}, 0.016 \mathrm{~mL} / \mathrm{min} / \mathrm{g}(0.004$ 0.059); and PVFES flux ${ }_{\mathrm{tot}}, 0.025 \mathrm{~mL} / \mathrm{min} / \mathrm{g}(0.004-0.092)$. For the 16 patients whose data were used for metabolite analysis, the mean (range) flux corr $_{\text {was }} 0.035 \mathrm{~mL} / \mathrm{min} / \mathrm{g}$ (0.010-0.067), and the mean (range) PVFES flux corr $_{\text {was }}$ $0.058 \mathrm{~mL} / \mathrm{min} / \mathrm{g}(0.011-0.18)$.

${ }^{18} \mathrm{~F}-\mathrm{FES}$ uptake measures were compared with qualitative ER uptake measured by IHC (Fig. 3). For ${ }^{18}$ F-FES uptake versus qualitative IHC results, $\rho=0.62(P=0.009)$ for PVSUV, $\rho=0.57(P=0.02)$ for PVFES flux $x_{\text {tot }}$, and $\rho=0.60$ $(P=0.01)$ for PVFES flux ${ }_{\text {corr }}$. For non-PV SUVs versus qualitative IHC results, $\rho=0.52(P=0.03)$. IHC indicated that 5 patients had ER-negative tumors and 12 patients had ER-positive tumors ( 3 with $2+$ immunostaining and 9 with $3+$ immunostaining); no patient in the series had $1+$ immunostaining. With the exception of one patient, all patients with ER-negative tumors had PVSUVs of less than 1.0, whereas patients with ER-positive tumors had values above 1.1. When an SUV of 1.1 was used to discriminate ERpositive and ER-negative tumors, there was $94 \%$ agreement (16/17 patients) for ${ }^{18} \mathrm{~F}-\mathrm{FES}$ uptake versus IHC results.

A single patient had an ER-negative core biopsy of the breast and a PVSUV above 1.1 (1.9). The original pathology report from her surgery indicated $3+$ immunostaining for ER expression analyzed by IHC and a high degree of heterogeneity. The results suggested that the portion of the tissue that was sampled for the present study was in a section of the tumor with a lower level of ER expression.

Similar plots were seen for PVFES flux ${ }_{\text {tot }}$, with ERnegative tumors having values of less than $0.01 \mathrm{~mL} / \mathrm{min} / \mathrm{g}$ (Fig. 3B), and for PVFES flux corr $_{\text {, with ER-negative tumors }}$ having values of less than $0.02 \mathrm{~mL} / \mathrm{min} / \mathrm{g}$ (Fig. 3C); again, the exception was the same patient with a higher SUV.

A patient found to be ER positive by IHC had a PVSUV of 1.1 and low PVFES flux ${ }_{\text {tot }}$ and PVFES flux corr $_{\text {values, }} 0.016$ and $0.021 \mathrm{~mL} / \mathrm{min} / \mathrm{g}$, respectively. Her tumor had a qualitative ER score of 3+ and an Allred score of 7. This patient had stopped hormone replacement medication approximately $1 \mathrm{wk}$ before her ${ }^{18} \mathrm{~F}-\mathrm{FES}$ scan. Her estradiol level was nor$\mathrm{mal}$, at $28 \mathrm{pg} / \mathrm{mL}$, at the time of the ${ }^{18} \mathrm{~F}-\mathrm{FES}$ scan. Although her hormone replacement medication may have competed with ${ }^{18} \mathrm{~F}-\mathrm{FES}$ uptake, this possibility is considered unlikely given the relatively low level of plasma estradiol at the time 

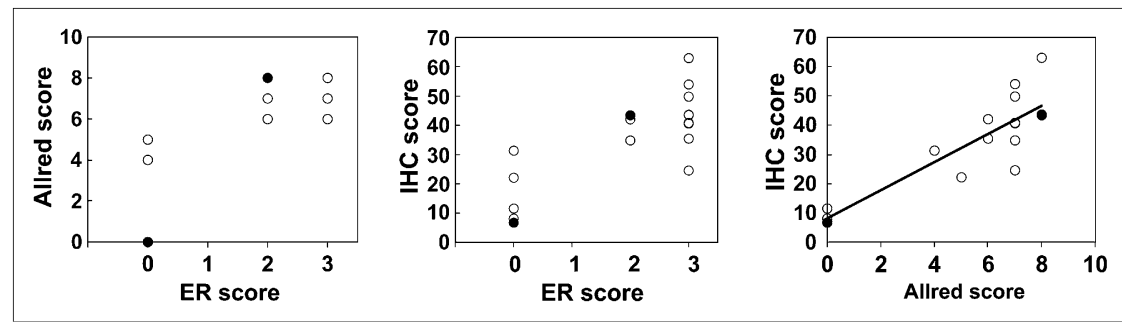

FIGURE 2. Comparison of qualitative and semiquantitative measures of $E R$ content by IHC of biopsy material. Left and center graphs compare qualitative ER scoring with Allred scoring and IHC index (Photoshop analysis). Treated patients are identified by closed circles.

of scanning. The difference between ${ }^{18} \mathrm{~F}$-FES PET results and in vitro assay results more likely reflects a difference between immune recognition (IHC) and functional binding, which has been reported for in vitro assays $(7,30,31)$.

${ }^{18} \mathrm{~F}$-FES uptake and semiquantitative Allred scores were compared as shown in Figure 4 . The $\rho$ values for ${ }^{18}$ F-FES uptake versus Allred scores (PVSUV, PVFES flux tot, and PVFES flux corr $)$ were $0.72(P=0.001), 0.61(P=0.01)$, and $0.60(P=0.01)$, respectively. For non-PV SUVs versus Allred scores, $\rho=0.65(P=0.005)$.

All 3 PV ${ }^{18}$ F-FES uptake measures showed significant correlations with the semiquantitative IHC index (Figs. 5A5C). As determined by Spearman analysis, $\rho=0.73(P<$ $0.001)$ for PVSUV, $\rho=0.63(P=0.007)$ for PVFES flux ${ }_{\text {tot }}$, and $\rho=0.57(P=0.02)$ for PVFES flux corr Correlation coefficients were not significantly different for the 3 measures when a linear bivariate fit was used: $r=0.64$ for PVSUV, $r=0.63$ for PVFES flux tot $_{\text {, and }} r=0.62$ for PVFES flux corr $P$ values were 0.005 for PVSUV, 0.007 for PVFES flux ${ }_{\text {tot }}$, and 0.01 for PVFES flux corr . For non-PV SUVs versus the IHC index, $r=0.35(P=0.17)$.

Excluding both the patient who had been on hormone replacement medication and the patient who had heterogeneous ${ }^{18} \mathrm{~F}$-FES uptake from the analysis resulted in slightly higher correlation coefficients. In the Allred score analysis, the Spearman correlation coefficients increased to 0.79 $(P<0.001), 0.75(P=0.001)$, and $0.72(P=0.004)$ for PVSUV, PVFES flux ${ }_{\text {tot }}$, and PVFES flux $x_{\text {corr }}$, respectively. In the IHC index analysis, the coefficients increased to 0.76 $(P<0.001), 0.73(P=0.002)$, and $0.64(P=0.01)$ for PVSUV, PVFES flux ${ }_{\text {tot }}$, and PVFES flux corr , respectively. Excluding these patients from the qualitative ER uptake and ${ }^{18} \mathrm{~F}$-FES uptake analysis increased the Spearman correlation coefficients to $0.76(P=0.001), 0.78 P<0.001)$, and $0.80(P<0.001)$ for PVSUV, PVFES flux $x_{\text {tot }}$, and PVFES flux ${ }_{\text {corr }}$, respectively. The results shown in Figures $1-5$ include all patients.
To examine the possible influence of short-term exposure to noninterfering breast cancer therapy, we excluded the 2 patients with short-term exposure to either aromatase inhibitors or chemotherapy and radiation therapy at the time of the ${ }^{18}$ F-FES PET scan and biopsy. Correlations between ${ }^{18} \mathrm{~F}-\mathrm{FES}$ uptake and IHC remained in this subset of patients $(n=15)$ : for PVSUV versus qualitative IHC, $\rho=$ $0.60(P=0.02)$; for PVSUV versus Allred scores, $\rho=0.66$ $(P=0.007)$; and for PVSUV versus the IHC index, $\rho=$ $0.68(P=0.006)$.

\section{DISCUSSION}

The purpose of the present study was to compare ${ }^{18} \mathrm{~F}-$ FES uptake with ER expression assayed in vitro by IHC with both qualitative and semiquantitative measures, complementing earlier studies (11) comparing ${ }^{18} \mathrm{~F}-\mathrm{FES}$ uptake with radioligand binding.

Three different methods of measuring ER expression by IHC were available on the same tissue slides for comparison with ${ }^{18} \mathrm{~F}$-FES uptake. Initial ER scoring was a qualitative assessment of the slides, and they were given scores of $0-3+$ on the basis of the intensity of the nuclear staining. In clinical practice, a score of $1+$ or higher is taken to indicate a tumor that may be sensitive to endocrine therapy (8). We also used a commonly known method of semiquantitative evaluation, the Allred method, which includes an 8-point scoring system based on the intensity and percentage of positively stained cells on a slide $(7,27)$. To take advantage of the quantitative nature of PET data with which the IHC results were compared, we applied a semiquantitative computerized analysis method (Photoshop) that we have used before (28). Although this method is not routinely used to assess ER expression in clinical practice, its reproducibility was excellent. The results of similar computerized analyses were previously tested and compared with the results of in vitro radioligand-binding assays for ER expression (32,33), like the method that we
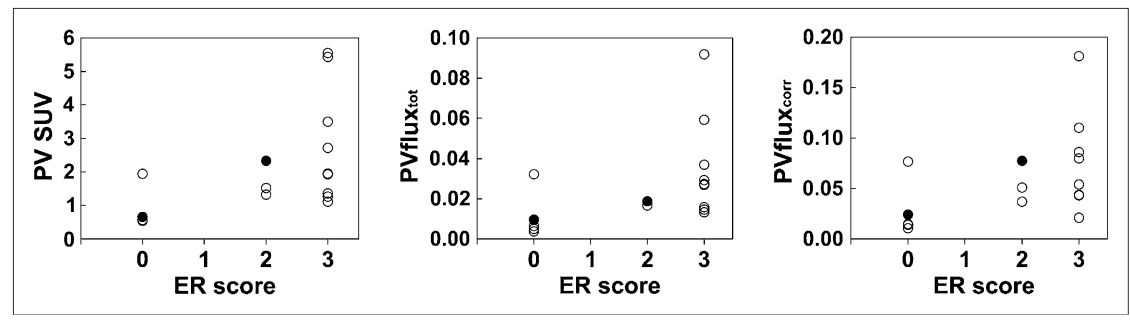

FIGURE 3. Comparison of ${ }^{18}$ F-FES uptake measures (PVSUV, PVFES flux tot, and PVFES flux corr ) with qualitative IHC results (ER expression scored as $0-3+$ ). Treated patients are identified by closed circles. 
FIGURE 4. Comparison of ${ }^{18} \mathrm{~F}-\mathrm{FES}$ uptake measures with Allred scores. Treated patients are identified by closed circles.
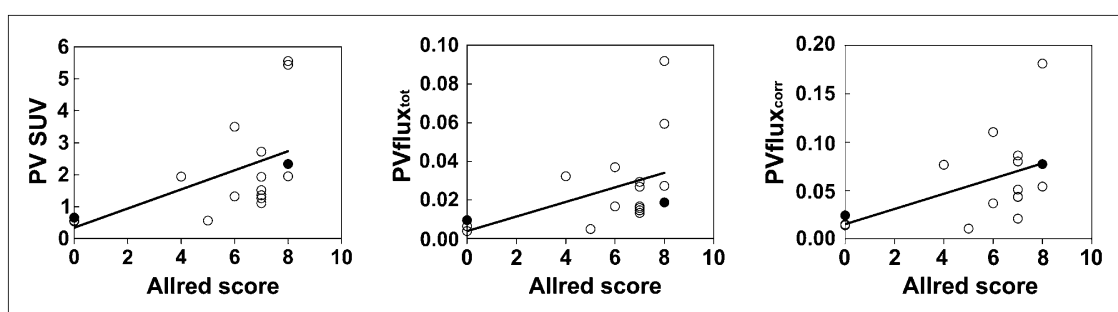

used (28). All methods of scoring ER expression by IHC correlated well with each other, as expected.

In 1988 (11), Mintun et al. reported good correlations between ${ }^{18} \mathrm{~F}-\mathrm{FES}$ uptake and in vitro ER concentrations determined by radioligand binding. In 1995, Dehdashti et al. (34) compared the separation of ER-positive and ER-negative cancers with ${ }^{18} \mathrm{~F}$-FES uptake in tumors and reported an overall agreement of $88 \%$. There has been a shift in clinical practice from using radioligand binding to using IHC for routine ER content analysis of breast cancers (15). Several studies included comparisons of in vitro radioligand-binding assays for ER expression with IHC assays for ER expression $(7,16$, $33,35)$. These studies showed that the agreement between the 2 methods ranged from $86 \%$ to $98 \%$. Our rate of agreement between the ${ }^{18} \mathrm{~F}$-FES uptake and IHC methods fell in this range.

In our earlier study comparing in vitro radioligand-binding assays and the IHC index, we found a linear correlation $(r)$ of 0.70 (28). Other studies including quantitative comparisons of radioligand-binding assays and IHC found similar IHC indices $(30,31,36-38)$. The correlation $(\rho)$ between ${ }^{18} \mathrm{~F}-\mathrm{FES}$ uptake and the IHC index that we found in the present study, 0.73 (linear correlation $[r]$ of 0.64 ), fell in the range of values reported in earlier studies comparing in vitro assays.

Our correlations, however, were lower than the correlation reported by Mintun et al. (11), $r=0.96$. Several factors could account for the differences in the results. There was a more uniform distribution of ER expression in the in vitro assays in our study than in the study of Mintun et al. (11); this difference could have altered the apparent correlation between the imaging and in vitro results. In addition, in the earlier study, in vivo radioligand binding $\left({ }^{18} \mathrm{~F}-\mathrm{FES}\right)$ was compared with in vitro radioligand binding; however, in the present study, in vivo radioligand binding was compared with the presence of ER epitopes determined by in vitro IHC. We would expect a lower correlation for our present study $\left({ }^{18} \mathrm{~F}-\mathrm{FES}\right.$ uptake versus in vitro IHC) than for the study of Mintun et al. (11) $\left({ }^{18} \mathrm{~F}-\mathrm{FES}\right.$ uptake versus in vitro radioligand binding), in accordance with studies comparing in vitro radioligand-binding assays and in vitro IHC assays (27, $30,31)$. Given the differences in sampling and quantification for in vivo imaging versus in vitro assays, we would not necessarily expect a perfect correlation in any instance. Importantly, in vivo measurement of ER expression by ${ }^{18} \mathrm{~F}-$ FES PET appeared to add predictive capability for the response to endocrine therapy, even in patients whose tumors were shown to express ER in in vitro assays (12-14).

We compared different measures of ${ }^{18} \mathrm{~F}-\mathrm{FES}$ uptake and ER concentrations, including PVFES flux $x_{\text {tot }}$ and PVFES flux $_{\text {corr }}$. In the present analysis, in distinction to our earlier, preliminary analysis $(18,39)$, the flux measures did not have a clear advantage over the simple SUV measure of ${ }^{18} \mathrm{~F}$-FES uptake. However, in studies of patients treated with agents that could alter estrogen levels or ${ }^{18} \mathrm{~F}-\mathrm{FES}$ clearance rates, the flux measures may be important to consider.

We identified a PVSUV threshold of 1.1 for distinguishing tumors determined to be ER positive and ER negative by IHC. Our earlier studies (14) and others (13) suggested that an ${ }^{18} \mathrm{~F}$-FES SUV of 1.5-2.0 (or greater) is predictive of a response to endocrine therapy. The IHC threshold for ER positivity is staining in $5 \%$ of nuclei or more. Other researchers, for example, Ogawa et al. (40), indicated that $10 \%$ staining may be an acceptable cutoff for predicting a response to endocrine therapy. Taken together, these results suggest that the quantitative threshold for the determination of endocrine responsiveness by ${ }^{18} \mathrm{~F}-\mathrm{FES}$ PET may be slightly higher than the threshold for correlation with standard IHC criteria for ER positivity.

There are inherent difficulties in comparing in vitro and in vivo parameters, including the size of the tissue sample. ER distribution may be heterogeneous throughout a lesion, and a tissue sample may not be representative of the entire lesion. This was likely the case with one of the outliers in the present study; for this patient, the initial ER status, determined from her surgery, was scored as $3+$, but the slides subsequently made for analysis were scored as ER negative.
FIGURE 5. Comparison of ${ }^{18}$ F-FES uptake measures with IHC index (Photoshop analysis). Treated patients are identified by closed circles.
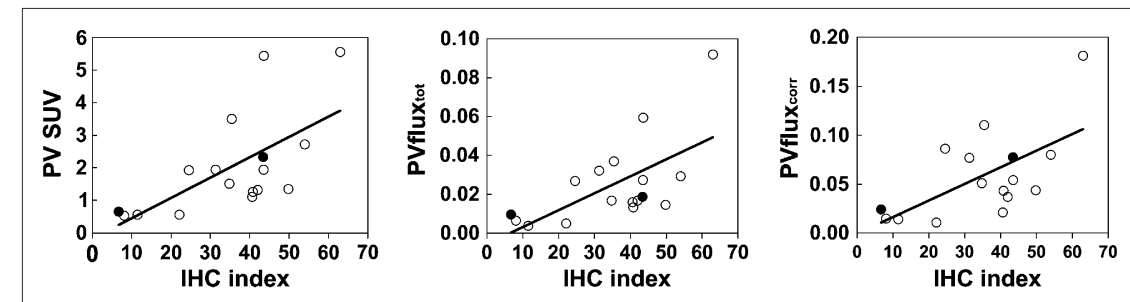
We are unable to conclusively explain an outlier with low ${ }^{18} \mathrm{~F}$-FES uptake in the group of patients found to have ERpositive tumors by IHC; this finding may simply reflect inherent differences between radioligand-binding assays for ER ( ${ }^{18}$ F-FES PET) and immunorecognition (IHC).

The present study has several limitations. Although the sample size $(n=17)$ is the largest used to date to compare ${ }^{18} \mathrm{~F}$-FES uptake with in vitro IHC analysis of ER expression, the patient population is still small. Additional samples would likely increase the significance of the correlation of quantitative measures of ER expression with ${ }^{18} \mathrm{~F}-\mathrm{FES}$ uptake.

The patient population was heterogeneous, with both male and female patients, newly diagnosed and recurrent breast cancers, a variety of types of previous therapy, tissue samples from different tumor sites, and ${ }^{18} \mathrm{~F}-\mathrm{FES}$ studies done at various intervals from the time of tissue sampling. Two patients had short-term exposure to agents with activity against breast cancer but not known to block estradiol binding to ER. This exposure did not appear to affect the correlation of ${ }^{18}$ F-FES uptake with IHC of the biopsy material; however, this feature was not rigorously tested.

The need for partial-volume correction of ${ }^{18} \mathrm{~F}$-FES uptake in smaller tumors is another limitation of the present study. Although recovery coefficients are necessary to account for the loss of signal attributable to finite spatial resolution, this correction is an approximate one based on an imperfect estimate of tumor size and likely adds variability to the ${ }^{18}$ F-FES uptake measures.

Despite these potentially confounding factors, the correlation between ${ }^{18} \mathrm{~F}$-FES uptake and ER expression remained significant and at the expected level.

\section{CONCLUSION}

${ }^{18}$ F-FES uptake correlated well with both qualitative and semiquantitative IHC measures of ER expression in biopsy material, suggesting that ${ }^{18} \mathrm{~F}$-FES uptake is a good indicator of regional ER expression in breast cancer when compared with the accepted clinical standard assay for ER expression, IHC. ${ }^{18}$ F-FES imaging cannot replace the use of in vitro ER measures, but it may be a useful tool for assessing the ER status of a patient's entire tumor burden, including metastatic sites or tumors that are difficult to biopsy. Future studies should examine the influence of hormonal therapy on ${ }^{18} \mathrm{~F}$-FES uptake and the response to therapy.

\section{ACKNOWLEDGMENTS}

The authors would like to thank Drs. Hannah Linden, Julie Gralow, and Robert Livingston for their help in referring patients to our study. The authors also thank the personnel at the University of Washington Radiology Department and PET Imaging Facility for technical support; the Seattle Cancer Care Alliance Breast Cancer Specialty Clinic for help with patient referrals; the personnel at Phenopath Laboratory
(Hadi Yazigi, Marilyn Skelly, and Lynn Goldstein) for help with tissue specimens; and Brenda Kurland and Mark Muzi for helpful comments. This work was supported by $\mathrm{NIH}$ grants P01CA42045, R01CA72064, and S10 RR17229.

\section{REFERENCES}

1. Jemal A, Siegel R, Ward E, et al. Cancer statistics, 2006. CA Cancer J Clin. 2006;56:106-130.

2. Honig SH, Swain SM. Hormonal manipulation in the adjuvant treatment of breast cancer. In: Devita VT, Hellman S, Rosenberg SA, eds. Important Advances in Oncology. Philadelphia, PA: J.B. Lippincott; 1993:103-123.

3. DeSombre ER, Thorpe SM, Rose C, et al. Prognostic usefulness of estrogen receptor immunocytochemical assays for human breast cancer. Cancer Res. 1986;46(suppl):4256s-4264s.

4. Kinsel LB, Szabo E, Greene GL, Konrath J, Leight GS, McCarty KS Jr. Immunocytochemical analysis of estrogen receptors as a predictor of prognosis in breast cancer patients: comparison with quantitative biochemical methods. Cancer Res. 1989;49:1052-1056.

5. Thorpe SM, Rose C, Rasmussen BB, et al. Steroid hormone receptors as prognostic indicators in primary breast cancer. Breast Cancer Res Treat. 1986; 7(suppl):S91-S97.

6. Allred DC, Bustamante MA, Daniel CO, Gaskill HV, Cruz AB Jr. Immunocytochemical analysis of estrogen receptors in human breast carcinomas: evaluation of 130 cases and review of the literature regarding concordance with biochemical assay and clinical relevance. Arch Surg. 1990;125:107-113.

7. Harvey JM, Clark GM, Osborne CK, Allred DC. Estrogen receptor status by immunohistochemistry is superior to the ligand-binding assay for predicting response to adjuvant endocrine therapy in breast cancer. $J$ Clin Oncol. 1999; 17:1474-1481.

8. Barnes DM, Harris WH, Smith P, Millis RR, Rubens RD. Immunohistochemical determination of oestrogen receptor: comparison of different methods of assessment of staining and correlation with clinical outcome of breast cancer patients. Br J Cancer. 1996;74:1445-1451.

9. Fisher ER, Anderson S, Dean S, et al. Solving the dilemma of the immunohistochemical and other methods used for scoring estrogen receptor and progesterone receptor in patients with invasive breast carcinoma. Cancer. 2005; 103:164-173.

10. Kiesewetter DO, Kilbourn MR, Landvatter SW, Heiman DF, Katzenellenbogen JA, Welch MJ. Preparation of four fluorine-18-labeled estrogens and their selective uptakes in target tissues of immature rats. J Nucl Med. 1984;25:1212-1221.

11. Mintun MA, Welch MJ, Siegel BA, et al. Breast cancer: PET imaging of estrogen receptors. Radiology. 1988;169:45-48.

12. Mortimer JE, Dehdashti F, Siegel BA, Katzenellenbogen JA, Fracasso P, Welch MJ. Positron emission tomography with $2-{ }^{18}$ F-fluoro-2-deoxy-D-glucose and $16 \alpha-{ }^{18} \mathrm{~F}$-fluoro- $17 \beta$-estradiol in breast cancer: correlation with estrogen receptor status and response to systemic therapy. Clin Cancer Res. 1996;2:933-939.

13. Mortimer JE, Dehdashti F, Siegel BA, Trinkaus K, Katzenellenbogen JA, Welch MJ. Metabolic flare: indicator of hormone responsiveness in advanced breast cancer. J Clin Oncol. 2001;19:2797-2803.

14. Linden HM, Stekhova SA, Link JM, et al. Quantitative fluoroestradiol positron emission tomography imaging predicts response to endocrine treatment in breast cancer. J Clin Oncol. 2006;24:2793-2799.

15. Chebil G, Bendahl PO, Idvall I, Fernö M. Comparison of immunohistochemical and biochemical assay of steroid receptors in primary breast cancer: clinical associations and reasons for discrepancies. Acta Oncol. 2003;42:719-725.

16. Kurosumi M. Significance of immunohistochemical assessment of steroid hormone receptor status for breast cancer patients. Breast Cancer. 2003;10: 97-104.

17. Mankoff DA, Tewson TJ, Eary JF. Analysis of blood clearance and labeled metabolites for the estrogen receptor tracer [F-18]-16 alpha-fluoroestradiol (FES). Nucl Med Biol. 1997;24:341-348.

18. Mankoff DA, Peterson LM, Petra P, et al. Factors affecting the level and heterogeneity of uptake of [F-18]fluoroestradiol (FES) in patients with estrogen receptor-positive (ER+) breast cancer [abstract]. J Nucl Med. 2002;43(suppl): 287P.

19. Mankoff DA, Dunnwald LK, Gralow JR, et al. Changes in blood flow and metabolism in locally advanced breast cancer treated with neoadjuvant chemotherapy. J Nucl Med. 2003;44:1806-1814.

20. Tewson TJ, Mankoff DA, Peterson LM, Woo I, Petra P. Interactions of $16 \alpha-$ $\left[{ }^{18} \mathrm{~F}\right]$-fluoroestradiol (FES) with sex steroid binding protein (SBP). Nucl Med Biol. 1999;26:905-913. 
21. DeGrado TR, Turkington TG, Williams JJ, Stearns CW, Hoffman JM, Coleman RE. Performance characteristics of a whole-body PET scanner. J Nucl Med. 1994;35:1398-1406.

22. Lewellen T, Kohlmyer S, Miyaoka R, Schubert S, Stearns C. Investigation of the count rate performance of the General Electric ADVANCE positron emission tomograph. IEEE Trans Nucl Sci. 1995;42:1051-1057.

23. Weinberg IN, Huang SC, Hoffman EJ, et al. Validation of PET-acquired input functions for cardiac studies. J Nucl Med. 1988;29:241-247.

24. Shields AF, Mankoff DA, Link JM, et al. Carbon-11-thymidine and FDG to measure therapy response. J Nucl Med. 1998;39:1757-1762.

25. Kohlmyer S, Vesselle HV, Miyaoka R, Kaplan M, Lewellen TK. Comparison of recovery coefficients for PET based on maximum and average TOI pixel values. Paper presented at: EANM Annual Meeting; September 2-6, 2000; Paris, France.

26. Huang SC. Anatomy of SUV: standardized uptake value. Nucl Med Biol. 2000;27:643-646.

27. Allred DC, Harvey JM, Berardo M, Clark GM. Prognostic and predictive factors in breast cancer by immunohistochemical analysis. Mod Pathol. 1998;11:155-168.

28. Lehr HA, Mankoff DA, Corwin D, Santeusanio G, Gown AM. Application of Photoshop-based image analysis to quantification of hormone receptor expression in breast cancer. J Histochem Cytochem. 1997;45:1559-1565.

29. Lehr HA, van der Loos CM, Teeling P, Gown AM. Complete chromogen separation and analysis in double immunohistochemical stains using Photoshopbased image analysis. J Histochem Cytochem. 1999;47:119-126.

30. Heubner A, Beck T, Grill HJ, Pollow K. Comparison of immunocytochemical estrogen receptor assay, estrogen receptor enzyme immunoassay, and radioligand-labeled estrogen receptor assay in human breast cancer and uterine tissue. Cancer Res. 1986;46(suppl):4291s-4295s.

31. McCarty KS Jr, Miller LS, Cox EB, Konrath J, McCarty KS Sr. Estrogen receptor analyses: correlation of biochemical and immunohistochemical methods using monoclonal antireceptor antibodies. Arch Pathol Lab Med. 1985;109:716-721.
32. Mofidi R, Walsh R, Ridgway PF, et al. Objective measurement of breast cancer oestrogen receptor status through digital image analysis. Eur J Surg Oncol. 2003;29:20-24.

33. Charpin C, Martin PM, De Victor B, et al. Multiparametric study (SAMBA 200) of estrogen receptor immunocytochemical assay in 400 human breast carcinomas: analysis of estrogen receptor distribution heterogeneity in tissues and correlations with dextran coated charcoal assays and morphological data. Cancer Res. 1988;48: 1578-1586.

34. Dehdashti F, Mortimer JE, Siegel BA, et al. Positron tomographic assessment of estrogen receptors in breast cancer: comparison with FDG-PET and in vitro receptor assays. J Nucl Med. 1995;36:1766-1774.

35. Ferrero-Pous M, Trassard M, Le Doussal V, Hacene K, Tubiana-Hulin M, Spyratos F. Comparison of enzyme immunoassay and immunohistochemical measurements of estrogen and progesterone receptors in breast cancer patients. Appl Immunohistochem Mol Morphol. 2001;9:267-275.

36. McClelland RA, Berger U, Miller LS, Powles TJ, Jensen EV, Coombes RC. Immunocytochemical assay for estrogen receptor: relationship to outcome of therapy in patients with advanced breast cancer. Cancer Res. 1986;46(suppl):4241s-4243s.

37. Hanna W, Mobbs BG. Comparative evaluation of ER-ICA and enzyme immunoassay for the quantitation of estrogen receptors in breast cancer. Am J Clin Pathol. 1989;91:182-186.

38. Esteban JM, Ahn C, Battifora H, Felder B. Predictive value of estrogen receptors evaluated by quantitative immunohistochemical analysis in breast cancer. Am J Clin Pathol. 1994;102(suppl):S9-S12.

39. Linden HM, Stekhova SA, Link JM, et al. HER2 expression and uptake of ${ }^{18} \mathrm{~F}-$ fluoroestadiol (FES) predict response of breast cancer to hormonal therapy [abstract]. J Nucl Med. 2004;45(suppl):85P.

40. Ogawa Y, Moriya T, Kato Y, et al. Immunohistochemical assessment for estrogen receptor and progesterone receptor status in breast cancer: analysis for a cut-off point as the predictor for endocrine therapy. Breast Cancer. 2004;11:267-275. 